\title{
Evaluation of two new methods for routine measurement of alkaline phosphatase isoenzymes
}

\author{
A P Day, S Saward, C M Royle, P D Mayne
}

\begin{abstract}
Aims: To evaluate the performance of two new methods for the analysis of alkaline phosphatase (ALP) isoenzymes designed for use in the routine chemical pathology laboratory: pre-incubation with neuraminidase before agarose electrophoresis; and selective precipitation of the bone isoenzyme with wheat germ agglutinin (WGA).

Methods: Serum samples from 39 patients were analysed. Seventeen were from patients with liver disease, eight from patients with bone disease, and 14 from patients with normal ALP activity and no evidence of liver or bone disease. The two new methods were compared with the established method, wheat germ agglutinin affinity electrophoresis.

Results: There was good correlation between the neuraminidase and WGA electrophoretic methods. The WGA precipitation method showed negative interference in the measurement of bone isoenzyme activity in samples containing biliary alkaline phosphatase. Both the new methods had advantages of speed and simplicity over the existing method, but cost per test was considerably higher.

Conclusions: The neuraminidase electrophoretic method is a satisfactory alternative to the WGA afinity electrophoretic method, although it is more expensive. The WGA precipitation method cannot be recommended for use with serum samples from patients with suspected liver disease.
\end{abstract}

The cause of increased serum alkaline phosphatase (ALP, EC 3.1.3.1) activity is usually apparent from clinical findings and other biochemical data. Under certain circumstances, however, it may be necessary to determine specific ALP isoenzyme activities-for example, when the tissue of origin is uncertain or to monitor treatment. ${ }^{1}$ The most common requirement is to measure liver and bone isoenzymes. Because these differ from each other only in minor differences in glycosylation, they are poorly separated by electrophoresis, therefore making accurate quantitation difficult. Resolution can be improved by presoaking cellulose acetate membranes in wheat-germ agglutinin (WGA), which binds to $\mathrm{N}$-acetylglucosamine and reduces the electrophoretic mobility of the bone isoenzyme. ${ }^{2}$ Brief preincubation of serum with neuraminidase (sialidase, EC 3.2.1.18), which removes sialic acid residues, also enhances separation by increasing the charge difference between the bone and liver isoenzymes. ${ }^{34}$

A simple method for quantitation of the bone isoenzyme has recently been described ${ }^{25}$ and marketed (Boehringer Mannheim Ltd, Lewes, England). ${ }^{67}$ This depends on the ability of WGA to precipitate bone ALP. Measurement of total and supernatant ALP activities permit the calculation of bone ALP activity by subtracting the difference.

We evaluated the performance of two methods against the standard method for the quantitation of bone ALP isoenzyme activity: WGA-affinity electrophoresis on cellulose acetate membrane ${ }^{2}$; agarose electrophoresis with neuraminidase (Helena Laboratories, Gateshead, England); and WGA precipitation (Boehringer Mannheim Ltd, Lewes, England).

\section{Methods}

Serum samples from 39 patients were analysed by each of the methods. Seventeen were from patients with liver disease, eight from patients with bone disease, and 14 from patients with normal ALP activity and no evidence of liver or bone disease. All samples were either stored at $4^{\circ} \mathrm{C}$ for up to four days before analysis or frozen at $-20^{\circ} \mathrm{C}$ and thawed for 48 hours at $4^{\circ} \mathrm{C}$ before analysis.

ALP activity was measured at $37^{\circ} \mathrm{C}$ using 4-nitrophenyl phosphate as substrate and 2-amino-2-methyl-1-propanol as buffer on an RA-1000 automatic analyser (Technicon Instruments Co Ltd, Basingstoke, England).

\section{WGA-AFFINITY ELECTROPHORESIS}

Serum $(1 \mu \mathrm{l})$ was electrophoresed at 250 volts for 60 minutes on cellulose acetate membrane (Sartorius GmbH, Gottingen, Germany) that had been presoaked in TRIS/Barbital/sodium barbital buffer, pH 8.8, 10.05 (Gelman Sciences Inc, Ann Arbor, USA). ALP activity was visualised by incubation for one hour at $37^{\circ} \mathrm{C}$ on an agar gel containing the chromogenic substrate 5-bromo-4-chloro-3indolyl phosphate (Sigma Chemical Co Ltd, Poole, England). After drying overnight, the isoenzymes were quantitated by reflectance densitometry. This allowed biliary and intestinal isoenzymes to be measured. Presoaking 
the membrane in a solution of WGA (Triticum Vulgaris, Sigma Chemical Co Ltd, Poole, England) $1 \mathrm{mg} / 100 \mathrm{ml}$ in running buffer for 10 minutes and then repeating the electrophoresis enhanced separation of the bone and liver isoenzymes sufficiently to permit densitometric quantitation. The detection limit for ALP activity by this method is $5-10 \mathrm{IU} / \mathrm{l}$.

\section{NEURAMINIDASE ELECTROPHORESIS}

Serum $(25 \mu \mathrm{l})$ was incubated with $5 \mu \mathrm{l}$ neuraminidase solution at room temperature for five minutes. Serum $(5 \mu \mathrm{l})$ both treated and untreated with neuraminidase was applied to preprepared agarose gels incorporating TRIS/ barbital/sodium barbital buffer $(\mathrm{pH}$ 8.6-9.0) and detergent (Helena Laboratories, Gateshead, England), and electrophoresed at 250 volts for 30 minutes. After electrophoresis ALP activity was visualised following incubation with 5-bromo-4-chloro-3-indolyl phosphate in the dark for 30 minutes at $45^{\circ} \mathrm{C}$. After drying at $70^{\circ} \mathrm{C}$, isoenzymes were quantitated by densitometry. The detection limit for ALP activity by this method is $3 \mathrm{IU} / 1$.

\section{WGA PRECIPITATION}

Serum $(100 \mu \mathrm{l})$ was mixed with $100 \mu \mathrm{l}$ precipitating solution $(5 \mathrm{~g} / 1 \mathrm{WGA}$ in acetate buffer, $\mathrm{pH} 4 \cdot 5,2 \%$ Triton X-100) (Boehringer Mannheim Ltd, Lewes, England). After standing for 30 minutes at room temperature, the mixture was centrifuged for two and a half minutes at $1000 \times g$. ALP activity was determined in both the neat serum and the supernatant. Bone ALP was calculated using

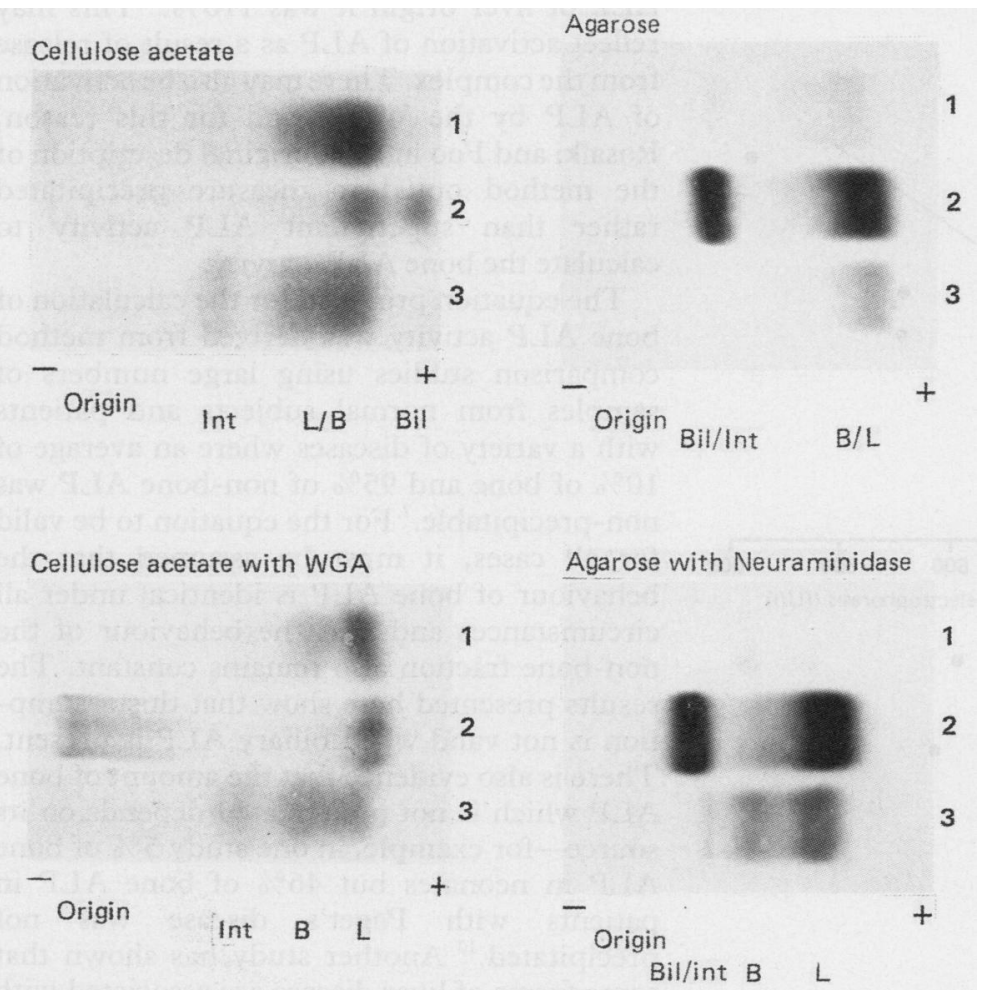

Figure 1 Separation of ALP isoenzymes by WGA-affinity cellulose acetate electrophoresis and by neuraminidase-agarose electrophoresis. Sample 1: liver and bone isoenzymes; sample 2: liver, bone and biliary isoenzymes; sample 3: liver, bone, and intestinal isoenzymes. the following formula, ${ }^{7}$ provided with the kit: Bone ALP $=1.118 \times$ total ALP $-2.35 \times$ supernatant ALP

Data were compared using the Wilcoxon two sample test. Linear regression was calculated by the least squares method and differences between the methods were assessed using the method of Altman and Bland. ${ }^{8}$

\section{Results}

Total ALP activity ranged from 44 to $965 \mathrm{IU} / 1$ (reference range 42-117 IU/1). Both electrophoretic methods resolved the bone and liver isoenzymes sufficiently to permit densitometric quantitation in all cases (fig 1). The range of ALP isoenzyme activities for each patient group by the WGA-affinity electrophoresis method is shown in the table. There was good correlation between the methods with no significant proportionate or constant bias between the results using the method of Altman and Bland.

Linear regression analysis of the results by WGA-affinity electrophoresis (x) compared with neuraminidase incubation $(y)$ gave the equation $\mathrm{y}=0.97 \mathrm{x}-0.01(\mathrm{n}=39, \mathrm{r}=0.997)$ for the bone isoenzyme; $y=0.99 x+0.78$ ( $n=39, r=0.993$ ) for the liver isoenzyme; and $y=0.84 x+15.01(n=22, r=0.965)$ for the biliary isoenzyme.

Total analytical and operator times for the WGA-affinity electrophoretic method were 15 hours and 60 minutes, respectively. Corresponding times for the neuraminidase-electrophoretic method were two hours and 45 minutes, respectively. Approximate cost per test of consumables was $£ 0 \cdot 70$ for WGAaffinity electrophoresis and $£ 3.65$ for neuraminidase-electrophoresis, assuming the optimal batch size of four samples.

As there was no significant difference between the two methods, the precipitation method was compared with the WGA-affinity electrophoretic method only.

Mean bone ALP activity by the precipitation method was $47 \%$ lower than by the electrophoretic method. Correlation between the methods was poor $(r=0.777)$ and nine of the samples had a calculated bone ALP activity of less than $0 \mathrm{IU} / 1$. These were all from patients with liver disease who had detectable biliary ALP activity (fig 2). In these samples there was, paradoxically, an increase rather than a decrease in serum ALP activity following addition of the precipitating reagent. Subgroup analysis showed that although there was no significant difference in bone ALP activity between normal subjects and patients with liver disease as measured by WGA-affinity electrophoresis, the WGA precipitation method gave results which were significantly lower in the liver disease group (table).

If samples containing biliary ALP were excluded the correlation between the two methods was good. For each pair of results, there was a good correlation between the difference and the average $(n=25 ; r=0.862$, $\mathrm{p}<0.01$ ) indicating a proportionate bias between the methods (fig 2B). The precipitation 
Comparison of $A L P$ isoenzyme activities in normal subjects, patients with liver disease, and patients with bone disease by WGA-affinity electrophoresis and WGA-precipitation (median and range)

\begin{tabular}{|c|c|c|c|c|c|}
\hline \multirow[b]{2}{*}{ Group } & \multirow[b]{2}{*}{$n=$} & \multicolumn{3}{|l|}{ Electrophoresis } & \multirow{2}{*}{$\frac{\text { Precipitation }}{\text { Bone ALP }(I U / l)}$} \\
\hline & & Bone ALP (IU/l) & Liver $A L P(I U / l)$ & Biliary $A L P(I U / l)$ & \\
\hline $\begin{array}{l}\text { Normal } \\
\text { Liver disease } \\
\text { Bone disease }\end{array}$ & $\begin{array}{r}14 \\
17 \\
8\end{array}$ & $\begin{array}{c}26(8-37) \\
20(6-69) \\
412(71-965)^{\star}\end{array}$ & $\begin{array}{c}39(15-65) \\
249(45-690) \star \\
35(0-164)\end{array}$ & $\begin{array}{l}0 \\
78(0-300)^{\star} \\
0\end{array}$ & $\begin{array}{c}17(8-39) \\
-7(-86-57)^{\star} \\
246(64-761)^{\star}\end{array}$ \\
\hline
\end{tabular}

${ }^{\star} p<0.05$ in comparison with normal group.

method gave lower bone ALP activity by $37 \cdot 1 \%$ (95\% confidence limits $25 \cdot 4-47 \cdot 1 \%$ ). The regression equation for WGA-affinity electrophoresis (x) compared with WGA-precipitation $(y)$ was $y=0.65 x-1.50(n=25$, $\mathbf{r}=0.971$ )

Analysis time by the precipitation method was about one hour 40 minutes, with an operator time of five minutes. Cost per test, assuming batch analysis of four samples was $£ 2 \cdot 13$.

\section{Discussion}

The methods in use for the quantitation of ALP isoenzymes are all, to a certain extent, unsatisfactory. Electrophoresis separates most of the isoenzymes but does not give adequate resolution of the liver and bone isoenzymes. The addition of $\mathrm{WGA}^{2}$ or neuraminidase ${ }^{3}$ improves resolution such that densitometric quantitation of these isoenzymes is possible. These techniques are time consuming, however, and the quality of the results obtained depends very much on the operator. The WGA precipitation method has been suggested as a quick and simple alternative method for the quantitation of bone ALP. ${ }^{6}$

Figure 2 Comparison
between bone ALP by
WGA-affinity
electrophoresis and by the
WGA-precipitation
method. ( $(0)$ samples with
no detectable biliary ALP
(O) samples containing
biliary $A L P$.
(A) Direct comparison.
Regression equation for
samples without biliary
ALP:y $=0.65 x-1.50$
$(n=25, r=0.971)$.
Line indicates $y=x$.
(B) Differences between
methods v average result.
For samples without
biliary ALP: $r=0.862$,
$n=25 ; p<0.01$.
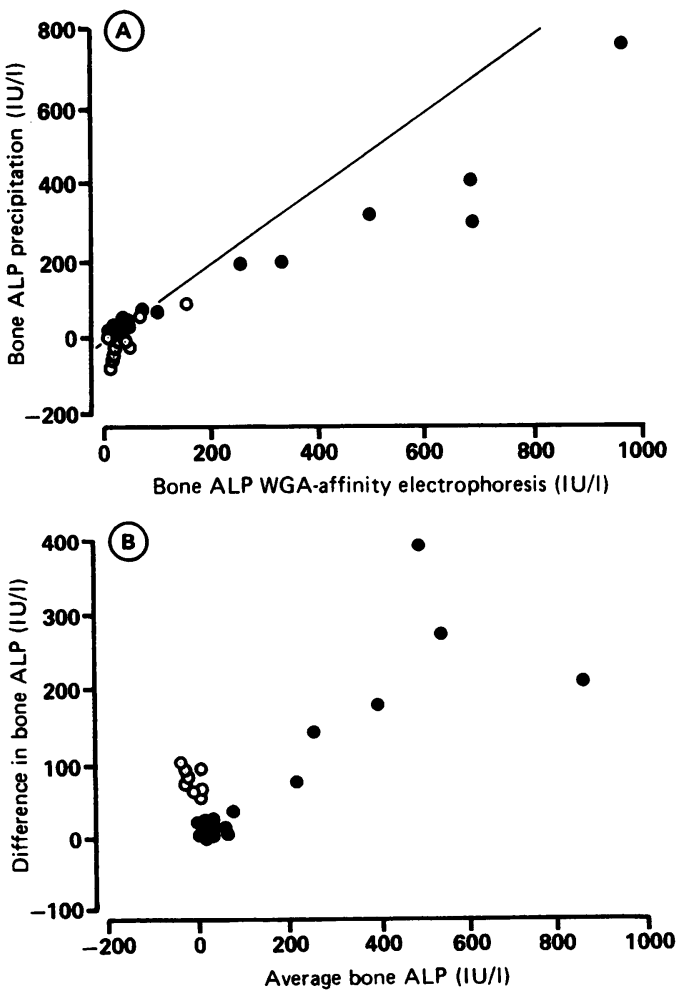

Both the WGA-affinity cellulose acetate and the neuraminidase-agarose electrophoretic methods gave good resolution with close agreement between the methods. Good separation of the liver and bone isoenzymes following pre-incubation with neuraminidase has also been reported using polyacrylamide gel electrophoresis, ${ }^{3}$ but the results are poor when cellulose acetate electrophoresis is used. ${ }^{9}$

A major problem was encountered using the WGA precipitation method with samples containing biliary ALP. This is precipitated by WGA. ${ }^{2}$ The inclusion of Triton X-100 in the precipitation solution dissociates the ALP from the complex and it then behaves exactly as liver ALP. ${ }^{2}$ This step is essential in the WGA precipitation method in order to prevent misclassification of biliary ALP as bone ALP. We observed an increase in total ALP activity in samples containing biliary ALP following the addition of the precipitating reagent. Although we did not redissolve ALP from the precipitates to calculate recovery, this finding agrees with analytical recovery data in the original description of this method where recovery in samples with increased bone ALP was $103 \%$ whereas in samples with increased ALP of liver origin it was $110 \% .^{2}$ This may reflect activation of ALP as a result of release from the complex. There may also be activation of ALP by the WGA, and for this reason, Rosalki and Foo in their original description of the method opted to measure precipitated rather than supernatant ALP activity to calculate the bone ALP activity.

The equation provided for the calculation of bone ALP activity was derived from method comparison studies using large numbers of samples from normal subjects and patients with a variety of diseases where an average of $10 \%$ of bone and $95 \%$ of non-bone ALP was non-precipitable. ${ }^{7}$ For the equation to be valid for all cases, it must be assumed that the behaviour of bone ALP is identical under all circumstances and that the behaviour of the non-bone fraction also remains constant. The results presented here show that this assumption is not valid when biliary ALP is present. There is also evidence that the amount of bone ALP which is not precipitated depends on its source-for example, in one study $5 \%$ of bone ALP in neonates but $46 \%$ of bone ALP in patients with Paget's disease was not precipitated. ${ }^{10}$ Another study has shown that some forms of liver disease are associated with production of precipitable ALP of hepatic origin which is not present in a high molecular weight complex but has a similar molecular 
mass to liver or bone ALP. ${ }^{5}$ Because it is the degree and nature of post-translational glycosylation which determines the ability of WGA to bind to the ALP molecule, ${ }^{11}$ it is conceivable that factors such as rate of production or the presence of abnormal glycosyltransferases may influence the final sugar chain structure. ${ }^{12}$

The WGA precipitation method may be adequate for determining whether the main source of increased serum ALP activity is from bone, but we have shown that it is inadequate for accurate quantitation in many cases. Raised biliary ALP activity results in negative interference with the measurement of bone ALP and the interaction of bone ALP with WGA probably varies with the type of patient under investigation.

Electrophoresis and densitometry remains the method of choice for accurate quantitation of serum ALP isoenzyme activity. The new neuraminidase-agarose electrophoretic method performs as well as the WGA-affinity cellulose acetate electrophoretic method and has the advantage of shorter analytical time, although the cost per test is considerably higher.
1 Moss DW. Alkaline phosphatase isoenzymes. Clin Chem 1982;28:2007-16.

2 Rosalki SB, Foo AY. Two new methods for separating and quantifying bone and liver alkaline phosphatase isoenzymes in plasma. Clin Chem 1984;30:1082-6.

3 Moss DW, Edwards RK. Improved electrophoretic resolution of bone and liver alkaline phosphatases resulting from partial digestion with neuraminidase. Clin Chim Acta partial digestion

4 Van Hoof VO, Lepoutre LG, Hoylaerts MF, Chevigne $R$, De Broe ME. Improved agarose electrophoretic method for separating alkaline phosphatase isoenzymes in serum. Clin Chem 1988;34:1857-62.

5 Behr W, Barnert J. Quantification of bone alkaline phosphatase in serum by precipitation with wheat-germ lectin: a simplified method and its clinical plausibility. Clin Chem 1986;32:1960-6.

6 Klein G, Bodenmuller H, Gerber M. Evaluation of a new photometric assay for the determination of human bone alkaline phosphatase. Clin Chem 1989;35:1089.

7 Rosalki S, Foo Y, Burlina A, et al. Evaluation of a new photometric Iso-ALP test kit in 4 clinical centres in Europe. Clin Chem 1990;36:1179.

8 Altman DG, Bland JM. Measurement in medicine: the analysis of method comparison studies. Statistician $1983 ; 32: 307-17$

9 Rosalki SB, Foo AY. Incubation with neuraminidase and affinity electrophoresis with wheat-germ lectin compared for separating and quantifying alkaline phosphatase. Clin Chem 1985;31:1198-200.

10 Miura M, Matsuzaki H. Evaluation of a new method for separating bone-type alkaline phosphatase. Ann Clin Biochem 1990;27:501-2.

11 Koyama I, Sakagishi Y, Komoda T. Different lectin affinities in rat alkaline phosphatase isozymes: multiple forms of the isozyme isolated by heterogeneities of sugar moieties. isozyme isolated by heterogen

12 Massoubre C, Verdier P, Meflah K, et al. Lectin binding to serum $5^{\prime}$-nucleotidase and alkaline phosphatase in liver disease. Clin Chim Acta 1990;191:87-92. 